\title{
Seminal Vesicle Angiosarcoma
}

National Cancer Institute

\section{Source}

National Cancer Institute. Seminal Vesicle Angiosarcoma. NCI Thesaurus. Code C161642.

An extremely rare angiosarcoma that affects the seminal vesicle. 